\title{
Characterization of thin gelatin hydrogel membranes with balloon properties for
} dynamic tissue engineering

\author{
Jepsen, Morten Leth; Nielsen, Line Hagner; Boisen, Anja; Almdal, Kristoffer; Dufva, Martin
}

Published in:

Biopolymers

Link to article, DOI:

10.1002/bip.23241

Publication date:

2019

Document Version

Peer reviewed version

Link back to DTU Orbit

Citation (APA):

Jepsen, M. L., Nielsen, L. H., Boisen, A., Almdal, K., \& Dufva, M. (2019). Characterization of thin gelatin hydrogel membranes with balloon properties for dynamic tissue engineering. Biopolymers, 110(1), [e23241]. https://doi.org/10.1002/bip.23241

\section{General rights}

Copyright and moral rights for the publications made accessible in the public portal are retained by the authors and/or other copyright owners and it is a condition of accessing publications that users recognise and abide by the legal requirements associated with these rights.

- Users may download and print one copy of any publication from the public portal for the purpose of private study or research.

- You may not further distribute the material or use it for any profit-making activity or commercial gain

- You may freely distribute the URL identifying the publication in the public portal 
Article type: Full paper

Characterization of thin gelatin hydrogel membranes with balloon properties for dynamic tissue engineering

Morten Leth Jepsen, Line Hagner Nielsen, Anja Boisen, Kristoffer Almdal, Martin Dufva*

MSc M. L. J., Dr. L. H. N., Prof. A. B., Prof. K. A., Dr. M. D.,

The Danish National Research Foundation and Villum Foundation's Center for Intelligent Drug Delivery and Sensing Using Microcontainers and Nanomechanics (IDUN), Department of Microand Nanotechnology, Technical University of Denmark, Ørsteds Plads 345C, 2800 Kgs. Lyngby, Denmark.

E-mail: Martin.Dufva@nanotech.dtu.dk

Keywords: hydrogels, rheology, gelatin, biomimetics

Cell or tissue stretching and strain are present in any in vivo environment, but is difficult to reproduce in vitro. Here, we describe a simple method for casting a thin (about $500 \mu \mathrm{m}$ ) and soft (about $0.3 \mathrm{kPa}$ ) hydrogel of gelatin and a method for characterizing the mechanical properties of the hydrogel simply by changing pressure with a water column. The gelatin is crosslinked with mTransglutaminase and the area of the resulting hydrogel can be increased up 13-fold by increasing the radial water pressure. This is far beyond physiological stretches observed in vivo. Actuating the hydrogel with a radial force achieves both information about stiffness, stretchability, and contractability, which are relevant properties for tissue engineering purposes. Cells could be stretched and contracted using the gelatin membrane. Gelatin is a commonly used polymer for hydrogels in tissue engineering, and the discovered reversible stretching is particularly interesting for organ modeling applications. 


\section{Introduction}

Hydrogels are polymeric solids swelled in substantial amounts of water, either of synthetic or natural origin. ${ }^{[1]}$ Hydrogels can be crosslinked in numerous ways, including enzymatic protein crosslinking, ${ }^{[2]}$ ionic gelation, ${ }^{[3]}$ self-assembly, ${ }^{[4]}$ and chemical crosslinking. ${ }^{[5]}$

In this work, we have utilized an enzymatically crosslinked gelatin hydrogel. Gelatin is derived from collagen, which is a major component of various connective tissues. Gelatin is denatured collagen fragments with a broad molecular weight distribution. ${ }^{[6]}$ Gelatin will structurally transit from a helix structure to a coil at a temperature around $36^{\circ} \mathrm{C}$, thereby phase transition from solid to liquid will occur. ${ }^{[6,7]}$ Cell cultures are normally grown at $37{ }^{\circ} \mathrm{C}$, and therefore the gelatin needs to be crosslink to be utilized as a hydrogel in a cell culture. The coil formations can be crosslinked by mTransglutaminase (mTG) resulting in a gelatin hydrogel that retain the coil structure of the gelatin at $37^{\circ} \mathrm{C}$. mTG crosslinked gelatin hydrogels are less tightly packed than a hydrogel resulting from cooling of dissolved gelatin forming a helix structured hydrogel. ${ }^{[8]}$ However, gelatin is just one out of many polymers which hydrogels can be made of. Depending on the type of polymer, the fabrication method, and the crosslinking method, hydrogels' physical and/or chemical properties can be tuned to suit various fields such as biomedicine, soft electronics, sensors, and actuators. ${ }^{[9-11]}$

Hydrogels have a potential use in the field of tissue engineering. Since the microenvironment of in vivo tissues is mechanically flexible, cells are exposed to mechanical forces of various durations, frequencies, and amplitudes. ${ }^{[12]}$ This work mainly focuses on mechanical flexibility in terms of a stretchable and contractible matrix. We use the term "dynamic surface" for this phenomenon, which resemble hollow organs e.g. the motions of alveoli in lungs. The mechanical properties of hydrogels are tunable e.g. the Young's modulus $(E)$ and can vary greatly. Hydrogels have been reported from very soft with $E=1.5 \mathrm{kPa}^{[13]}$ to very stiff with $E>600 \mathrm{kPa} \cdot{ }^{[14]}$ By tuning the stiffness of hydrogel it is possible to tune them for the specific tissue of interest. 
Previous publications have presented various approaches to achieve hydrogels with twodimensional (2D) dynamic surface behaviors. One approach is to expose cells to equibiaxial stretch by functionalizing hydrogels on top of a stretchable material such as polydimethylsiloxane (PDMS $)^{[15]}$ or stretching coated parafilm or PDMS. ${ }^{[16-19]}$ However, permeable hydrogels supported by neither synthetic materials nor impermeable materials have closer resemblance with connective tissue more. Unsupported dynamic cell-laden hydrogels have been achieved by adding cells in a collagen hydrogel into a three-dimensional (3D) printed mesh of a stretchable poly(ethylene glycol) (PEG)-alginate-nanoclay hydrogel. ${ }^{[20]}$ Other approaches include integration of magnets that could be used for stretching the hydrogel ${ }^{[21,22]}$ or by seeding cells on top of a hydrogel for uniaxial stretch by moving the ends of the hydrogel apart. ${ }^{[23]}$ However, all these approaches solely achieve a 2D stretch whereas we aim for achieving a 3D expandable hydrogel balloon.

Mechanical characterization of hydrogels is not straight forward due to their low Young's modulus making them difficult to clamp and most mechanical characterization equipment is optimized for measurements in the range of $\mathrm{MPa}$ and $\mathrm{GPa}^{[24]}$. Furthermore, hydrogels in the swollen state have rubbery mechanical behavior, and the Young's modulus of hydrogels is dependent on the degree of swelling. ${ }^{[25,26]}$ Thus, hydrogels should be fully immersed (completely covered by a relevant medium) during characterization. Characterizations performed in air rather than liquid typically reduces the swelling due to evaporation. One approach to ensure the conditions of the hydrogel is by letting it swell before measuring, ${ }^{[27]}$ but it can dry during measurements e.g. in a rheometer.

One method of mechanical characterization methods preformed with immersed and fully swelled hydrogels is indentation, where a probe is pressed into and retracted from the material. ${ }^{[24]}$ However, using indentation does not give information about stretchability nor contractability of the hydrogel. A method of stretching fully immersed hydrogels using a stainless steel ball has previously been 
published. ${ }^{[26]}$ By measuring the central displacement caused by the stainless steel ball to the hydrogel membrane Young's modulus could be determined. However, by this approach, the force is centered to the middle of a hydrogel. Here, we present a method of stretching gelatin hydrogels fully submerged where the pressure comes from a liquid rather than a solid object causing a close to spherical inflation of the hydrogel. The stiffness, stretch, contraction, and durability were characterized. Furthermore, an epithelial and an endothelial cell line was stretched using the presented method.

\section{Materials and Methods}

$3 D$ printing of gelatin hydrogel membrane holder: 3D designs were drawn using Fusion 360 (version 1.28.2, Autodesk, San Rafael, California, United States) and print files were exported as STL format and processed in PreForm (version 2.12.0, Formlabs, Somerville, Massachusetts, United States). The 3D printed holder was designed with a small ring at the opening tube for the membrane to attach when casted (Figure S1, S2, Supporting Information). All prints was done using a a Formlabs Form 2 3D printer (Formlabs, Somerville, Massachusetts, US).

Prints for demonstration of the stretch principle were printed in Clear resin (Clear V2 FLGPCL02, Formlabs, Somerville, Massachusetts, United States) with a layer thickness of $0.1 \mathrm{~mm}$ followed by cleaning in isopropanol two times for $10 \mathrm{~min}$ and ultraviolet (UV) crosslinked for 60 min at room temperature. Prints for cell cultures were printed in Dental SG resin (Dental SG DGOR01, Formlabs, Somerville, Massachusetts, United States) with a layer thickness of $0.05 \mathrm{~mm}$, and were subsequently cleaned as described above. Support structures were cutoff, and the structures were UV crosslinked for $60 \mathrm{~min}$ at $60{ }^{\circ} \mathrm{C}$ and autoclaved at $121{ }^{\circ} \mathrm{C}$. 
Casting of expandable hydrogel membrane: For casting expandable hydrogel membranes, 3D prints with a $10 \mathrm{~mm}$ diameter opening were dipped in a solution, if not stated otherwise, consisting of $\mathrm{mTG}$ (5 U/mL) (Ajinomoto Activa T1, Hamburg, Germany) and gelatin (5 \% (w/v)) (Fluka, 48723) in phosphate buffered saline (PBS) (D8537, Sigma, St Louis, United States) and incubated for $30 \mathrm{~min}$ for cell cultures, and $120 \mathrm{~min}$ for expansion experiments, both at $37^{\circ} \mathrm{C}$ (Supplementary Figure 2). Incidentally, it has been shown that gelatin film display highest elongation when crosslinked at $35^{\circ}$ C. ${ }^{[28]}$ Stock solution of $\mathrm{mTG}$ was made by dissolving $\mathrm{mTG}$ in PBS in final concentration of 15 $\mathrm{U} / \mathrm{mL}$. Stock solution of gelatin was made by dissolving gelatin in PBS to a final concentration of $7.5 \%(\mathrm{w} / \mathrm{v})$. For sterilisation purposes, chloroform was added to the gelatin solution with final concentration of $0.5 \%(\mathrm{v} / \mathrm{v})$ of the stock solution. Whereas $\mathrm{mTG}$ was sterile filtered using a $0.45 \mu \mathrm{m}$ filter prior to use. The solutions were subsequently mixed in ratios giving $5 \%$ gelatin $(\mathrm{w} / \mathrm{v}))$ and $\mathrm{mTG} 5 \mathrm{mTG} \mathrm{U} / \mathrm{mL}$ in final concentrations. The procedure was the same for $2.5 \%(\mathrm{w} / \mathrm{v})$ and $7.5 \%(\mathrm{w} / \mathrm{v})$ gelatin membrane, but with either a $3.75 \%(\mathrm{w} / \mathrm{v})$ or $11.25 \%(\mathrm{w} / \mathrm{v})$ stock solution of gelatin, respectively.

For calculation of the membrane thickness, four membrane samples were weighed and found to be $45.18 \pm 3.32 \mathrm{mg}(\mathrm{n}=4$, mean \pm SD). The thickness was calculated from the weight, the radius of the gel $(5 \mathrm{~mm})$, and specific gravity of $5 \%$ gelatin $(1.012)^{[29]}$ :

$$
h=\frac{V}{\pi r^{2}}(1)
$$

where, $h$ is the thickness, $V$ is the volume, and $r$ is the radius.

Rheological measurements: Measurements were performed using a Discovery Hybrid Rheometer 2 (TA instruments, New Castle, Delaware, United States) with a $40 \mathrm{~mm}$ parallel plate and a steel Peltier plate. A mixture of gelatin/mTG $(500 \mu \mathrm{L})$ was added to the Peltier plate set at $37^{\circ} \mathrm{C}$. When the parallel 
plate was at the geometry gap of $250 \mu \mathrm{m}$, mineral oil (M5310, Sigma, St Louis, United States) was added around the plate to avoid evaporation. For investigation of gelation time, a strain of $1.5 \%$ was used with a frequency of $5 \mathrm{~Hz}$. For measurements of oscillation frequency dependence, a logarithmic sweep was done with a strain of $1.5 \%$ with frequencies increasing from $0.02 \mathrm{~Hz}$ to $15.92 \mathrm{~Hz}$. For measurements of strain breaking point, a logarithmic sweep was performed with a frequency of $5 \mathrm{~Hz}$ while increasing the strain from 0.1 to $3000 \%$.

Shear modulus was calculated by:

$$
G^{2}=G^{\prime 2}+G^{\prime 2}
$$

where, $G$ is the shear modulus, $G$ ' is the storage modulus, and $G$ ' 'is the loss modulus. Since $G$ ' 'is close to $0 G \approx G^{\prime} \approx 360 \mathrm{~Pa}$. A Poisson's ratio of 0.5 was assumed, as the hydrogel is regarded as an incompressible material. Therefore, the Young's modulus is calculated by:

$$
E=2 G(1+\mu)(3)
$$

where, $E$ is Young's modulus, $G$ is the shear modulus, and $\mu$ is Passion's ratio giving a Young's modulus of $\sim 1080 \mathrm{~Pa}$.

Stretch of fully immersed hydrogels: The hydrogel membranes were placed in a beaker of PBS in a $37{ }^{\circ} \mathrm{C}$ heating oven and more PBS (colored with food dye) was added in steps of $200 \mu \mathrm{L}$. Pictures were taken using a Samsung WB32F compact camera. The pictures were subsequently fitted to a circle using a Plugin for Fiji ${ }^{[30]}$ (Contact_Angle.jar, version 2006/12/07, Marco Brugnara) from which the radius and length of the base of the circle segment was obtained and normalized to a ruler in the beaker (Figure S5, Supporting Information).

From this, the chord length, $a$, of a circular segment and radius, $R$, of the circle were obtained. From this the central angle, $\theta$, was calculated by ${ }^{[31]}$ :

$$
\theta=2 \sin ^{-1}\left(\frac{a}{2 R}\right)(4)
$$


From which the arc length, $s$, could be calculated by ${ }^{[31]}$ :

$$
s=R \theta
$$

However, when the circle segment of interest is the majority of the circle the arc length was calculated by:

$$
s=2 \pi R-R \theta
$$

The equations (5) and (6) gives the arc length of the small circle segment, which is the stretched length $(s)$. The stretch ratio $(A)$ is defined as the ratio of $\mathrm{s}$ to the original diameter of the gel:

$$
A=\frac{s}{\text { Original diameter }}(7)
$$

The stretch ratio and the force acting, calculated from the centimeter of water of the water column, on the membrane were fitted into the following equation for equibiaxial stretching ${ }^{[32]}$ :

$$
f=\mu_{r}\left[A^{\alpha_{r}-1}-A^{-\left(1+2 \alpha_{r}\right)}\right](8)
$$

Where $f$ is the force per unit undeformed area, $A$ is the stretch ratio, and $\mu_{r}$ and $\alpha_{r}$ are constants. The fitting was done in RStudio (Version 1.0.136, RStudio, Inc., Boston, Massachusetts, United States) using a nonlinear least squares fit.

From the fitted $\mu_{r}$ and $\alpha_{r}$, the shear modulus was calculated by ${ }^{[32]}$ :

$$
G=\frac{\mu_{r} \alpha_{r}}{2}(9)
$$

where, $G$ is the shear modulus. $G$ was inserted in Equation (3) to obtain the Young's modulus.

The surface area of the spherical cap, was calculated by ${ }^{[33]}$ :

$$
S_{\text {cap }}=2 \pi R H(10)
$$

where, $S_{\text {cap }}$ is the surface area, $R$ is the radius, and $H$ is the height of the circle segment, which is calculated by ${ }^{[33]}$ :

$$
H=R \pm \sqrt{R^{2}-b^{2}}
$$


where, $H$ is the height of the circle segment, $R$ is the radius of the circle, and $b$ is the radius of the base of the circle segment. When the circle segment of interest was the majority of the circle, $\sqrt{R^{2}-b^{2}}$ is added to $R$, when the circle segment is the minority $\sqrt{R^{2}-b^{2}}$ is subtracted.

When having the surface area from equation (10) was used to calculated the stretch area ratio with the following equation:

$$
\text { Stretch area ratio }=\frac{S_{\text {cap }}}{\text { Original area }}(12)
$$

Fatigue test and breaking strength of hydrogel membranes: Hydrogels prepared as described were tested for fatigue by adding $4 \mathrm{~mL}$ of PBS and removing and re-adding $1 \mathrm{~mL}$ of the solution 100 times. A picture was taken after every 10 pipetting steps.

The breaking strength was defined as the last point at which the membrane was intact and addition of $200 \mu \mathrm{L}$ buffer more resulted in rupture of the membrane.

Cell growth on stretchable hydrogel membranes: Caco-2 cells (ATCC® HTB-37 ${ }^{\mathrm{TM}}$, LGC Standards GmbH, Wesel, Germany) p9-14 were grown in T-75 cell culture flaks (Starstedt, Nümbrecht, Germany) along with $13 \mathrm{~mL}$ of Dulbecco's Modified Eagle's medium (DMEM) - high glucose (Sigma-Aldrich Denmark A/S Broendby, Denmark) with heat inactivated fetal bovine serum (FBS) $(20 \%(\mathrm{v} / \mathrm{v}))($ Biowest SAS, Nuaillé, France), non-essential amino acids (NEAA)(1 \% (v/v))(Gibco, Fisher Scientific, Slangerup, Denmark), and Penicillin-Streptomycin (P/S) (100 U/mL penicillin and $100 \mu \mathrm{g} / \mathrm{mL}$ Streptomycin) (Sigma-Aldrich Denmark A/S Broendby, Denmark).

EA.hy926 cells (ATCC® CRL-2922 ${ }^{\mathrm{TM}}$, LGC Standards GmbH, Wesel, Germany) p20-25 were grown in T-75 cell culture flaks (Starstedt, Nümbrecht, Germany) along with $13 \mathrm{~mL}$ of DMEM - high glucose (Sigma-Aldrich Denmark A/S Broendby, Denmark) with heat inactivated FBS (20 \% 
(v/v))(Biowest SAS, Nuaillé, France), and P/S (100 U/mL penicillin and $100 \mu \mathrm{g} / \mathrm{mL}$ Streptomycin) (Sigma-Aldrich Denmark A/S Broendby, Denmark).

The cell culture medium was changed every other day and upon 80-90\% confluency, the cells were split. The cells were washed with PBS followed by incubation with trypsinethylenediaminetetraacetic acid (EDTA) solution (0.5 g/L porcine trypsin and $0.2 \mathrm{~g} / \mathrm{L}$ EDTA-4Na) (Sigma-Aldrich Denmark A/S Broendby, Denmark). Once the cells were detached, trypsin-EDTA were inhibited by addition of cell culture medium, and a sample was taken for counting the cells (counted with NucleoCounter NC-200). The rest of the suspension was collected and spun for 5 min (800 rounds per minute (rpm)), followed by washing the cells in PBS and spun again for 5 min (800 rpm). The cells were seeded $(50,000$ cells in $500 \mu \mathrm{L})$ on top of a casted stretchable hydrogel membranes with $1 \mathrm{~mL}$ cell medium below. The cells were allowed to adhere to the membrane overnight at $37^{\circ} \mathrm{C}$ and $5 \% \mathrm{CO}_{2}$ before stretching by removal of basolateral medium.

Microscopy on stretch hydrogels: Microscopy images were captured using a Zeiss Axio Observer Z1 inverted microscope (Carl Zeiss MicroImaging GmbH, 37081 Gottingen, Germany), using an EC Plan-Neofluar 5x/0.16 Ph1 M27 or a LD Plan-Neofluar 40x/0.6 Korr M27 objective (Carl Zeiss MicroImaging GmbH, 37081 Gottingen, Germany). Initially $1000 \mu \mathrm{L}$ medium was below the hydrogel membrane. For stretching and contraction $600 \mu \mathrm{L}$ was removed and re-added.

Scanning electron microscopy on dried stretch hydrogel membranes: Gelatin membranes in a 3D printed holder (Supplementary Figure 1b) were inflated by air and dried for $66 \mathrm{~h}$ before being mounted for scanning electron microscopy micrographs (SEM). The samples were scanned using a Hitachi TM3030 tabletop microscope (Hitachi High-Technologies Europe GmbH, Krefeld, Germany) in low vacuum mode at $15 \mathrm{kV}$ operating voltage. 
Statistics: The data are presented as number of repetitions (n), mean \pm standard deviation (SD). Calculations were done using RStudio (Version 1.0.136, RStudio, Inc., Boston, Massachusetts, United States) and Microsoft Excel (Version 15.41, Microsoft Office, Seattle, Washington, United States).

\section{Results and Discussion}

\subsection{Fully immersed Young's modulus characterization of the gelatin hydrogel membrane}

The presented method allows for characterization of a stretched hydrogels while being fully immersed in a relevant medium, which to the authors' knowledge has not been previously presented. In the presented setup a thin gelatin membrane was casted in a 3D printed holder (Figure S1, S2, Supporting Information). The gelatin membrane attaches to the 3D printed holder during the casting and crosslinking reaction. Initial test showed that the gelatin membrane was firmly attached to the edges and did not easily detach when exposed to water pressure. In fact, overpressure resulted in rupture of the gelatin membrane with the remaining gelatin parts sticking to the rim of the 3D printed holder. We conclude that the gelatin membrane forms a very robust bond with the $3 \mathrm{D}$ printed holder.

The thin gelatin membrane was inflated as a balloon by increasing the pressure on it with a water column (Figure 1a). The hydrogel membrane could be stretched as a water column above the membrane exposed it to an increased pressure (Figure 1a). As the pressure increased an approximately spheroidal shaped inflated membrane was created which could be fitted as a circle (Figure S5, Supporting Information). From this, the stretch of the membrane was determined as a stretch ratio from equation (7), as described in Materials and Methods. Data points were plotted as pressure versus stretch ratio (Figure 1b). We fit both our small and large deformation data with equation (8) ${ }^{[32]}$ describing inflation in terms of stretch as a result of increased pressure on a circular 
balloon-like membranes into a spheroidal shape. For this the hydrogels are assumed as isotropic elastic solids, hence their strain only depends on stretch on the principal axis $(\mathrm{X}, \mathrm{Y}$, and $\mathrm{Z})$ relative to the 'ground state'. We use the term elasticity as a description of the ability of a material to return to its original shape after deformation, which in principle can be of any magnitude.

Equation (8) is derived ${ }^{[32]}$ assuming an incompressible material, thus, taking the thinning of the film at large deformation into account. From this fit the shear modulus can be extracted from equation (9). The result of fitting is in principle the shear modulus, G, which is valid at all deformations. We have chosen to convert our numbers into Young's moduli to facilitate comparison with literature data although from the point of view of our measurements this conversion is not needed since we exclusively deal with shear moduli. The Young's modulus was calculated by inserting the obtained shear modulus in equation (3), thereby characterizing the stiffness of a hydrogel while it was fully immersed.

When stretching a $5 \%$ gelatin hydrogel crosslinked for 2 hours with $\mathrm{mTG}$, there was a lag phase at low pressure $(<150 \mathrm{~Pa})$ where the membrane was stretched only slightly. Above about 200 $\mathrm{Pa}$, the pressure affected the stretching in linear fashion (Figure 2a). This inflation profile resembles inflation of a balloon where the polymer at a certain pressure allows inflation and further stretch requiring a smaller increase in pressure relative to the proportionally larger pressure increase required during the initial stetching phase.

A $5 \%$ gel could be stretched up to 4 -fold in the length direction (increase of the perimeter of the gel). Eventually, the membrane would burst and the breaking strength was calculated to be $0.49 \pm 0.09 \mathrm{kPa}(\mathrm{n}=9$, mean $\pm \mathrm{SD})$. A $7.5 \%$ gelatin hydrogel could be stretched but did not break even when filling up the test setup completely with water, hence the breaking strength is $>0.89 \mathrm{kPa}$. (Figure 2a). A $2.5 \%$ membrane could be extended but no measurements were possible as no water column 
was formed due to the softness of this membrane (Figure S3f, Supporting Information). Instead, the gel expanded until it bursted.

The Young's modulus for $5 \%$ gelatin hydrogel membranes was $0.35 \pm 0.08 \mathrm{kPa}(\mathrm{n}=9$, mean $\pm \mathrm{SD}$ ), calculated as described above, whereas for $7.5 \%$ membranes, the Young's modulus was $0.75 \pm 0.31 \mathrm{kPa}(\mathrm{n}=5$, mean $\pm \mathrm{SD})$ (Figure $2 \mathrm{~b})$. A storage modulus of $\sim 0.26 \mathrm{kPa}$, corresponding to a Young's modulus of $\sim 0.78 \mathrm{kPa}$, was measured for the $5 \%$ gelatin hydrogel in a Discovery Hybrid Rheometer 2 (measured $2 \mathrm{~h}$ after it had been crosslinked at $37^{\circ} \mathrm{C}$ inside the rheometer) (Figure S6a, Supporting Information). However, after about $8 \mathrm{~h}$ the storage modulus settled on $\sim 0.36 \mathrm{kPa}$ (Figure S6a, Supporting Information) corresponding to a Young's modulus of $\sim 1.08 \mathrm{kPa}$. In summary, the stretch measurement gave a Young's modulus of $0.35 \mathrm{kPa}$, whereas measurements with a rheometer gave a higher Young's modulus of $0.78 \mathrm{kPa}$.

Previously, measurements of samples of $5 \%$ gelatin and comparable amounts of mTG as used here, have shown Young's modulus in the range of $\sim 6 \mathrm{kPa}$ measured on a Solid Analyzer at $37^{\circ} \mathrm{C}$ without having the sample immersed. ${ }^{[34]}$ Others have shown Young's modulus of $12.4 \mathrm{kPa}$ of $5 \%$ gelatin hydrogels where the measurements were performed at room temperature. ${ }^{[35]}$ The lower modulus measured in the stretch setup (Figure 2) compared to the rheometer and Solid Analyzer is believed to be caused by swelling of hydrogels in the immersed state.

Stretching by pressure from a liquid or a gas rather than a stainless-steel ball allows for functionalization of the hydrogel surface without disturbing the functionalization when inflating and/or stretching the surface. The inflation of the hydrogels in the presented setup does not result in a perfect sphere due to attachment at the rim. This causes inhomogeneous deformation with equibiaxial stretching at the pole and planar elongation at the rim. ${ }^{[36]}$ The characteristics of the 
inflation resemble the inflation of a balloon, where in the presented setup the lumen of the hydrogel balloon acts as a dynamic surface.

\subsection{Characteristics of the gelatin hydrogel membrane}

The hydrogel membrane was measured to be $0.58 \pm 0.04 \mathrm{~mm}(\mathrm{n}=4$ mean $\pm \mathrm{SD})$ thick (Figure $3 \mathrm{a})$. The thickness of the presented hydrogel membrane is comparable to the previously published stretchable hydrogels which range from $0.13 \mathrm{~mm}^{[22]}$ to $3 \mathrm{~mm}^{[37]}$. The hydrogel could be reversibly expanded and contracted (Figure 3b) which is important if e.g. the lung is to be modelled. The gel typically was destroyed during handling where a pipette easily ruptures it. Stretch ratios of above 20 times have been published ${ }^{[37]}$ for one-dimensional (1D) stretches. By contrast, we observed stretch ratio of about 4 for a 5\%, however, the area was stretchable up to 13-folds (Figure S6b, Supporting information). It is however unclear if $2 \mathrm{D}$ stretching compares to $3 \mathrm{D}$ stretching. A two to four-fold stretching in the length scale and 13-fold stretching in the area is more than sufficient for modulating tissues (see below). Furthermore, the stretching was shown to been reversible with gelatin membranes being able to be stretched and contracted at least 100 times. These tests were done by pipetting water up and down forcing the gelatin membrane to oscillate between 2.8 and 3.2 stretch ratio (data not shown).

It was found that the hydrogel was inflatable when adding PBS (Figure 3b) and $70 \%(\mathrm{v} / \mathrm{v})$ ethanol (Figure 3c), and when applying an air pressure (Figure 3c) indicating that the composition of the medium is not important for the expansion. Expansion of air as well as other liquids is essential for modelling various tissues like joints, skin, lungs, heart and intestine etc.

Food dye could diffuse through the hydrogel gel over time. After 16h, the dye has diffused completely into the receiving volume of the beaker. Interestingly, after an overnight incubation, the water column of the buffer solution decreased to a level where the membrane was only slightly inflated (Figure 3d). This indicates that water has diffused through the gel into the receiving beaker. 
It is likely that this is due to the higher water pressure on the upper side of the gel due to the applied water column.

Other shapes and sizes of the gelatin hydrogel membranes were also feasible e.g. a larger membrane with a diameter of $18 \mathrm{~mm}$ and a $16 \times 16 \mathrm{~mm}$ square could easily be formed (Figure S3, Supporting Information). Moreover, the membrane inflated with air could be dried in its inflated shape (Figure S4a-c, Supporting Information), and once dried, SEM revealed a smooth surface of the gelatin hydrogel membrane (Figure S4d, e, Supporting Information).

Prior published stretchable hydrogels rely on complicated casting procedures such as purification of proteins, ${ }^{[27,38]} 3 \mathrm{D}$ printing, ${ }^{[20]}$ integration of magnets,,${ }^{[21,22]}$ nanopatterned hydrogels, ${ }^{[23]}$ nanocomposition of clay nanosheets, ${ }^{[13,14]}$ or a mixture of natural and synthetic polymers. ${ }^{[37]}$ Whereas, the presented method only requires mixing gelatin and mTG. This reduces time and cost and diminishes the technical difficulties in when casting stretchable hydrogel. Furthermore, mTG is nontoxic, biocompatible, and is the most studied enzyme in protein-based crosslinked hydrogels in tissue engineering. ${ }^{[35]}$ The mechanical behavior of gelatin crosslinking with $\mathrm{mTG}$ is dependent on the temperature at which the crosslinking is performed.

\subsection{Stretch of cell culture on a gelatin hydrogel membrane}

The gelatin membranes was subsequently used to study stretching of non-confluent cell layers. Nonconfluent cell layer was used to easily see if cells where stretched and move relative to each other. Caco-2 (an epithelial colorectal adenocarcinoma cell line), a cell line commonly used for intestine modelling, ${ }^{[39]}$ cells were seeded on the membranes in a 3D printed holder placed in a well plate (Figure S2, Supporting Information). Once the Caco-2 cells had attached to the membrane, they were stretched by changing the water pressure on the outside of the 3D printed membrane holder, (Figure 4a). In the initial state, the cell islands were close to each other (Figure $4 b$ ), and by removal of medium 
from the basolateral side on the 3D printed holder, the cell islands were stretched apart (Figure 4c). The cell islands got closer together again by re-addition of the basolateral medium as the membrane re-contracted (Figure 4d). Observing cell islands being stretched apart would not be possible when stretching a hydrogel in a rheometer with a microscope extension, since the cells are on top of the hydrogel and the rheometer pushes a cylinder onto the sample.

The CRL-2922 human umbilical vein endothelial cell line was used to show morphological changes to cells when exposed to stretching of the gelatin membrane. CRL-2922 cells were allowed to attach to the gelatin membrane and the cells display typical endothelial cell morphology (Figure 4e) before stretching. Stretching the gelatin membrane resulted in stretching of the adhered endothelial cells (Figure 4f). This resulted in cells that got further elongated (one example is indicated by the black circle) or had one end detached from the surface (white circle). Cells that did not adhere well to the gelatin from start did not show stretching. CRL-2922 cells that kept adhering to the gelatin membrane could be re-contracted when contracting the gelatin membrane (Figure 4g, black marked cell). Also, cells that got partly released (white circle) showed contraction when the gelatin membrane was contracted. Hence, the gelatin membrane can be utilized to investigate individual cell reactions to stretching.

The mechanically stimulated deformation of the membrane is reversible; thus the membrane setup is suitable for mimicry of dynamic physiological movements. Moreover, the membrane mimics the extracellular matrix, since gelatin resembles collagen and connective tissue found in the body. ${ }^{[1,40]}$ In addition, epithelial bending is essential for lumen morphogenesis, ${ }^{[41]}$ hence the presented method could be applicable for tissue engineering of hollow organs. One lumen morphogenic pathway is apical constriction by which the epithelial cells undergo apical shrinkage while keeping their volume constant. The apical constriction must be followed by an increase of the height of the cells and/or basal expansion. ${ }^{[41,42]}$ By stretching the cells on the presented gelatin 
hydrogel, it would be possible to cause both apical constriction and basal expansion. One such organ is the lung where it has been shown that apical constriction initiate new branching of lung tissue in chicken embryos. ${ }^{[43]}$ The lung tissue is exposed to cyclic stretch and contraction with a rate of $\sim 12$ cycles/min in humans, as a result of breathing. ${ }^{[4]}$ For rat lungs, a $40 \%$ increase in the surface area of the alveolar epithelial basement membrane was found at $100 \%$ total lung capacity. ${ }^{[45]}$ The presented gelatin hydrogel is suitable as an alveolar epithelial basement membrane substrate, since it can easily be stretched $40 \%$ (Figure $2 \mathrm{a}$ ) and subsequently contracted with cells attached (Figure 4d). However, the increase of $40 \%$ surface area was achieved by increasing the pressure transpulmonary pressure from $2 \mathrm{cmH}_{2} \mathrm{O}(\sim 0.2 \mathrm{kPa})$ to $25 \mathrm{cmH}_{2} \mathrm{O}(\sim 2.5 \mathrm{kPa}),{ }^{[45]}$ comparing this to the pressure required to stretch the gelatin hydrogel (Figure 2a) the gelatin hydrogel is much softer. On the other hand, the alveolar epithelial basement membrane is supported by a layer of epithelial cells, endothelial cells, and elastic fibers making the alveoli strong. ${ }^{[46,47]}$ Therefore, the in vivo tissue has more support than then presented gelatin hydrogel. The gelatin hydrogel can be tuned in strength by increasing the percentage of gelatin as was shown with $7.5 \%$ gelatin (Figure 2). Normally, a barrier consists of a confluent cell layer. However, in the current setup it was not possible to change medium without disturbing the cells or rupturing the membrane. Thus, it is not possible to grow the cells for longer periods. Future studies will involve medium changes using pumps that gently can control expansion and contraction during medium changes to minimize stretching cell layer more than what is physiologically relevant.

\section{Conclusion}

We present a method of measuring Young's modulus on soft hydrogels fully immersed in buffer only by changing the pressure of a water column on top of the hydrogel. The Young's modulus measurements from the presented method were lower than that of benchmark techniques most likely 
caused by the hydrogel being fully immersed during measurements. We present a soft gelatin hydrogel with a Young's modulus around $0.35 \mathrm{kPa}$. The gelatin hydrogel composed of a natural polymer that is biocompatible and an extracellular matrix was utilized as a matrix for cells and stretching and contraction of the cells were done. The surface area of the gelatin hydrogel can be stretched by a factor of 13 , which is beyond what is physiologically relevant. Moreover, the surface of the gelatin hydrogel can be functionalized with mammalian cell line cells for tissue engineering purposes.

\section{Supporting information}

Supporting Information available from the Wiley Online Library or from the author.

\section{Acknowledgements}

The authors would like to acknowledge the Danish National Research Foundation (DNRF122) and Villum Fonden (Grant No. 9301) for Intelligent Drug Delivery and Sensing Using Microcontainers and Nanomechanics (IDUN). Line Hagner Nielsen would like to acknowledge Danish Research Council for Technology and Production (FTP), Project DFF 4004-00120B for financial support.

MLJ contributed with design of and execution of experiment and analysis of data and writing of manuscript. LHN, AB, KA, and MD contributed with design of experiments and writing the manuscript.

\section{References}

[1] K. Almdal, J. Dyre, S. Hvidt, O. Kramer, Polym. Gels Networks 1993, 1, 5.

[2] T. Heck, G. Faccio, M. Richter, L. Thöny-Meyer, Appl. Microbiol. Biotechnol. 2013, 97, 461.

[3] W. R. Gombotz, S. Wee, Adv. Drug Deliv. Rev. 1998, 31, 267. 
[4] L. E. R. O’Leary, J. A. Fallas, E. L. Bakota, M. K. Kang, J. D. Hartgerink, Nat. Chem. 2011, 3,821 .

[5] W. E. Hennink, C. F. van Nostrum, Adv. Drug Deliv. Rev. 2012, 54, 13.

[6] M. Djabourov, J. Leblond, P. Papon, J. Phys. 1988, 49, 319.

[7] H. Boedtker, P. Doty, J. Phys. Chem. 1954, 58, 968.

[8] J. S. Suwandi, R. E. M. Toes, T. Nikolic, B. O. Roep, Clin. Exp. Rheumatol. 2015, 33, 97.

[9] Y. S. Zhang, A. Khademhosseini, Science 2017, 356, eaaf3627.

[10] S. Lin, H. Yuk, T. Zhang, G. A. Parada, H. Koo, C. Yu, X. Zhao, Adv. Mater. 2016, 28, 4497.

[11] I. El-Sherbiny, M. Yacoub, Glob. Cardiol. Sci. Pract. 2013, 38, 316.

[12] S. Ahadian, R. Civitarese, D. Bannerman, M. H. Mohammadi, R. Lu, E. Wang, L. Davenport-Huyer, B. Lai, B. Zhang, Y. Zhao, S. Mandla, A. Korolj, M. Radisic, Adv. Healthcare Mater. 2017, 1700506.

[13] K. Haraguchi, T. Takehisa, Adv. Mater. 2002, 14, 1120.

[14] Y. Hu, Z. Du, X. Deng, T. Wang, Z. Yang, W. Zhou, C. Wang, Macromolecules 2016, 49, 5660 .

[15] L. Casares, R. Vincent, D. Zalvidea, N. Campillo, D. Navajas, M. Arroyo, X. Trepat, Nat. Mater. 2015, 14, 343.

[16] D. Wang, Y. Xie, B. Yuan, J. Xu, P. Gong, X. Jiang, Integr. Biol. 2010, 2, 288.

[17] A. Livne, E. Bouchbinder, B. Geiger, Nat. Commun. 2014, 5, 3938.

[18] X. Shi, L. Li, S. Ostrovidov, Y. Shu, A. Khademhosseini, H. Wu, ACS Appl. Mater. Interfaces 2014, 6, 11915.

[19] C. P. Ursekar, S. K. Teo, H. Hirata, I. Harada, K. H. Chiam, Y. Sawada, PLoS One 2014, 9, e90665.

[20] S. Hong, D. Sycks, H. F. ai Chan, S. Lin, G. P. Lopez, F. Guilak, K. W. Leong, X. Zhao, Adv. Mater. 2015, 27, 4035.

[21] Y. Li, G. Huang, M. Li, L. Wang, E. L. Elson, T. Jian Lu, G. M. Genin, F. Xu, Sci. Rep. 2016, 6, 19550.

[22] Y. Li, G. Huang, B. Gao, M. Li, G. M. Genin, T. J. Lu, F. Xu, NPG Asia Mater. 2016, 8, e238.

[23] J. Deng, C. Zhao, J. P. Spatz, Q. Wei, ACS Nano 2017, 11, 8282.

[24] M. L. Oyen, Int. Mater. Rev. 2014, 59, 44.

[25] K. S. Anseth, C. N. Bowman, L. Brannon-Peppas, Biomaterials 1996, 17, 1647.

[26] M. Ahearne, Y. Yang, A. J. El Haj, K. Y. Then, K.-K. Liu, J. R. Soc. Interface 2005, 2, 455.

[27] N. Annabi, S. M. Mithieux, P. Zorlutuna, G. Camci-unal, A. S. Weiss, A. Khademhosseini, Biomaterials 2013, 34, 5496.

[28] F. Liu, H. Majeed, J. Antoniou, Y. Li, Y. Ma, W. Yokoyama, J. Ma, F. Zhong, Food Hydrocoll. 2016, 58, 20.

[29] J. J. Emery, W. D. L. Finn, K. W. Lee, In Evaluation of Relative Density and Its Role in Geotechnical Projects Involving Cohesionless Soils; American Society for Testing and Materials, 1973; pp. 182-194.

[30] J. Schindelin, I. Arganda-Carreras, E. Frise, V. Kaynig, M. Longair, T. Pietzsch, S. Preibisch, C. Rueden, S. Saalfeld, B. Schmid, J. Y. Tinevez, D. J. White, V. Hartenstein, K. Eliceiri, P. Tomancak, A. Cardona, Nat. Methods 2012, 9, 676.

[31] E. W. Weisstein, "Circular Segment". From MathWorld--A Wolfram Web Resource. http://mathworld.wolfram.com/CircularSegment.html (accessed: July 2018).

[32] R. W. Ogden, Proc. R. Soc. A Math. Phys. Eng. Sci. 1972, 326, 565.

[33] E. W. Weisstein, "Spherical Cap." From MathWorld--A Wolfram Web Resource. 
[34] R. Pimentel C., S. K. Ko, C. Caviglia, A. Wolff, J. Emnéus, S. S. Keller, M. Dufva, Acta Biomater. 2017, 65, 174.

[35] G. Yang, Z. Xiao, X. Ren, H. Long, H. Qian, K. Ma, Y. Guo, PeerJ 2016, 4, e2497.

[36] O. Hassager, S. B. Kristensen, J. R. Larsen, J. Neergaard, J. Nonnewton. Fluid Mech. 1999, 88,185 .

[37] J.-Y. Sun, X. Zhao, W. R. K. Illeperuma, O. Chaudhuri, K. H. Oh, D. J. Mooney, J. J. Vlassak, Z. Suo, Nature 2012, 489, 133.

[38] M. A. Gonzalez, J. R. Simon, A. Ghoorchian, Z. Scholl, S. Lin, M. Rubinstein, P. Marszalek, A. Chilkoti, G. P. López, X. Zhao, Adv. Mater. 2017, 29, 1.

[39] P. Artursson, K. Palm, K. Luthman, Adv. Drug Deliv. Rev. 2001, 46, 27.

[40] K. Y. Lee, D. J. Mooney, Chem. Rev. 2001, 101, 1869.

[41] A. Navis, C. M. Nelson, Semin. Cell Dev. Biol. 2016, 55, 139.

[42] E. J. Pearl, J. Li, J. B. A. Green, Philos. Trans. R. Soc. B Biol. Sci. 2017, 372, 20150526.

[43] H. Y. Kim, V. D. Varner, C. M. Nelson, Development 2013, 140, 3146.

[44] C. M. Waters, E. Roan, D. Navajas, Compr. Physiol. 2012, 2, 1.

[45] D. J. Tschumperlin, S. S. Margulies, J. Daniel, J. Appl. Physiol. 1999, 86, 2026.

[46] A. L. Kierszenbaum, L. L. Tres, In Histology and Cell Biology: An Introduction to Pathology; Elsevier, 2012; pp. 387-414.

[47] W. F. Boron, E. L. Boulpaep, In Medical Physiology; Elsevier, 2012; pp. 685-699.

Figure 1. Schematic of the experimental setup. a) Gelatin hydrogel membrane (gray) in a 3D printed holder (black) was inflated as the pressure increases from a watercolumn (light gray). b) Corresponding plot of pressure versus stretch ratio. By fitting the data to equation (8) the Young's modulus can be extracted.

Figure 2. Immersed stretch of gelatin hydrogel membrane. a) Pressure as a function of stretch ratio for experiments with individual fits to equation (8). b) The corresponding Young's modulus extracted from the immersed stretch experiments.

Figure 3. Characteristics of the stretchable gelatin hydrogel. a) Gelatin hydrogel membrane. b) Reversible expansion by addition and removal of $1 \mathrm{~mL}$ PBS on top of the gelatin membrane in a beaker with PBS. c) Gelatin hydrogel membrane expanded with $1 \mathrm{~mL} 70 \%$ ethanol on top in a beaker with $70 \%$ ethanol and membrane expanded by addition of air pressure from a syringe. $d$ ) Difussion of blue food dye from $1 \mathrm{~mL}$ PBS food dye mixture thourgh the gelatin hydrogel into a beaker with PBS after $30 \mathrm{~min}$ and after $16 \mathrm{~h}$.

Figure 4. Streching of Caco-2 and CRL-2922 on gelatin hydrogel membrane. a) By removing medium from the outside of the inset with the membrane the membrane is stretch and by re-adding medium the membrane recontracts. b) Caco- 2 cells on gelatin hydrogel membrane after 1 day incubation. Scale bar: $200 \mu \mathrm{m}$. c) Caco- 2 cells on gelatin hydrogel membrane stretched by removing $600 \mu \mathrm{L}$ medium basolaterally. Scale bar: $200 \mu \mathrm{m}$. d) Caco-2 cells on gelatin hydrogel membrane re-contracted by addition of $600 \mu \mathrm{L}$ medium basolaterally. Scale bar: $200 \mu \mathrm{m}$. e), CRL2922 cells on gelatin hydrogel membrane after 1 day incubation. Scale bar: $20 \mu \mathrm{m}$. f), CRL-2922 cells on gelatin hydrogel membrane stretched by removing $600 \mu \mathrm{L}$ medium basolaterally. Scale bar: 
$20 \mu \mathrm{m}$. g) CRL-2922 cells on gelatin hydrogel membrane re-contracted by addition of $600 \mu \mathrm{L}$ medium basolaterally. Scale bar: $20 \mu \mathrm{m}$. 
a

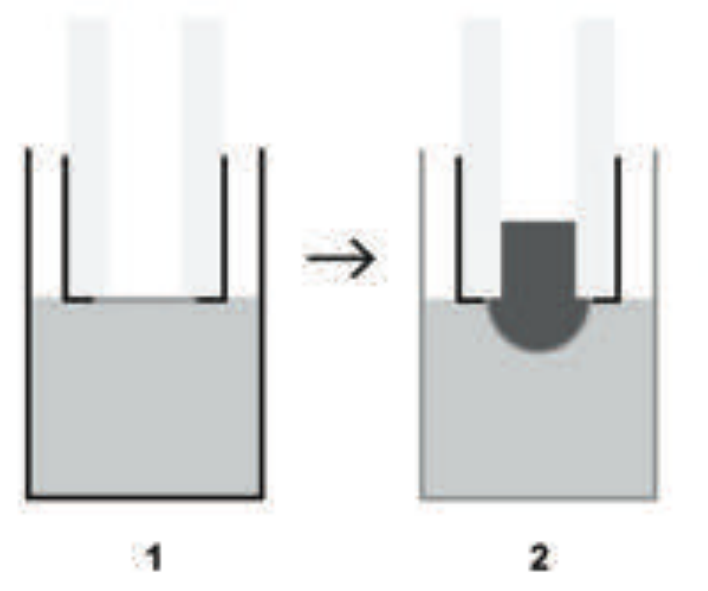

b

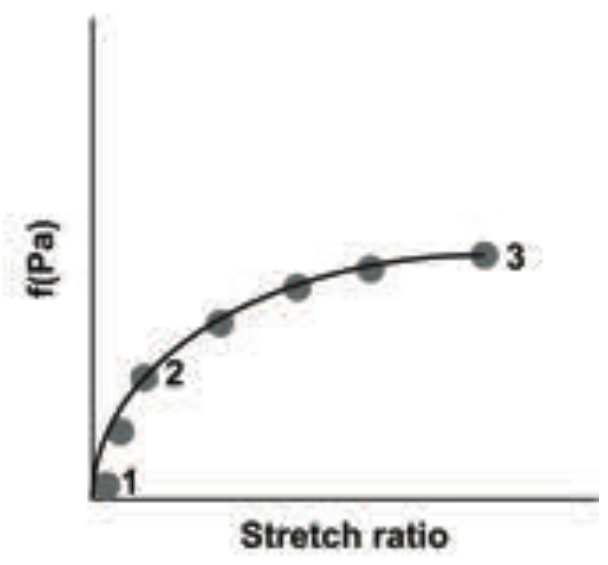


a

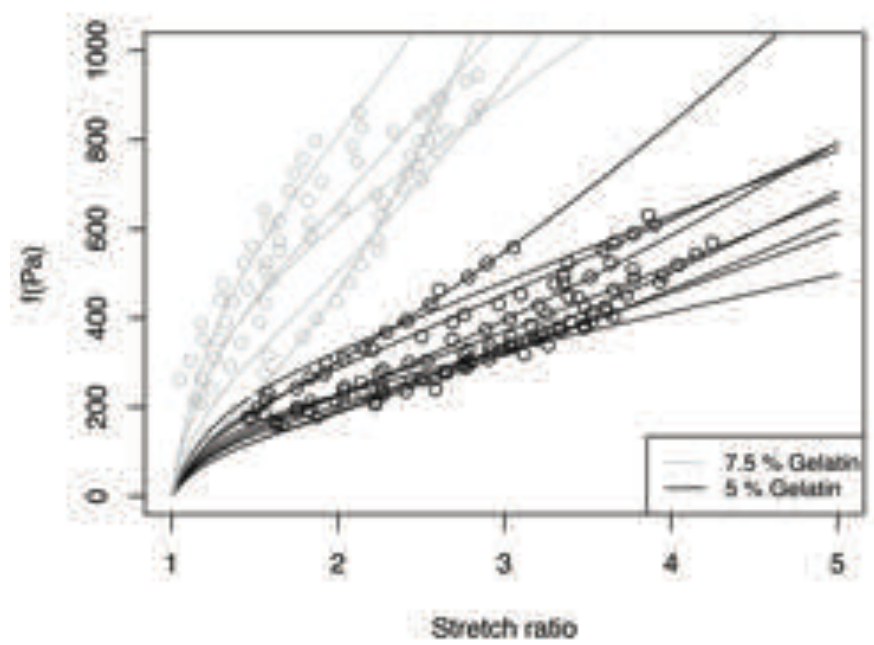

b

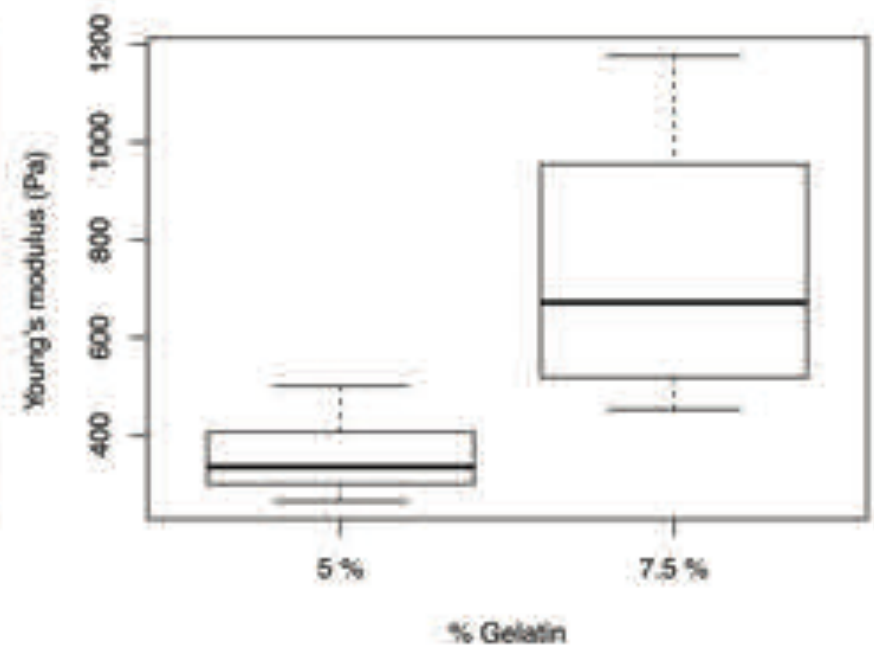



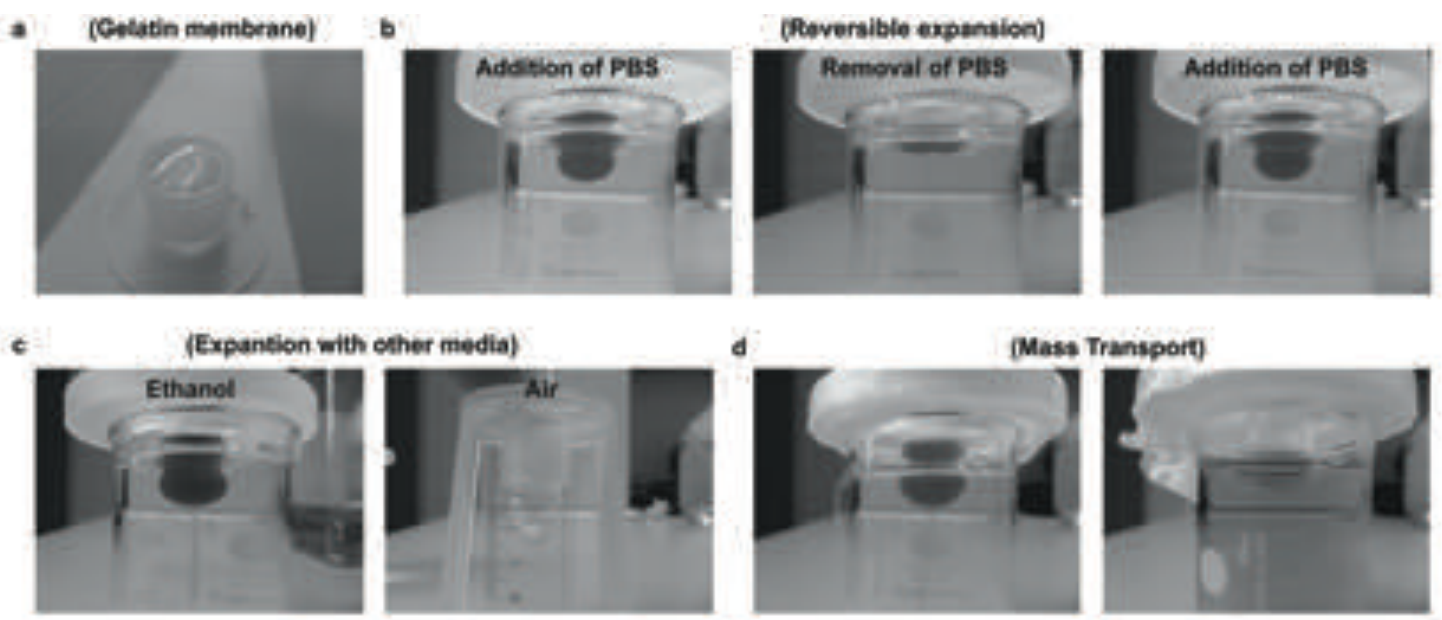
(A)

(B)

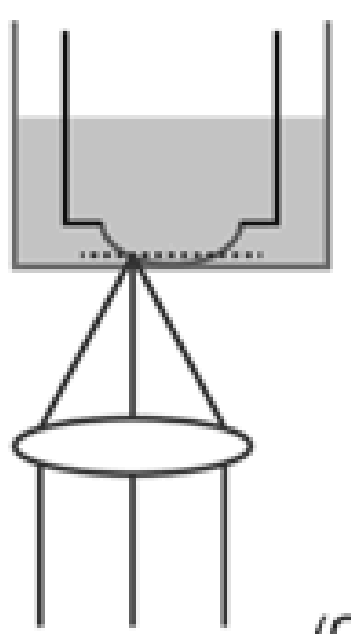

(C)

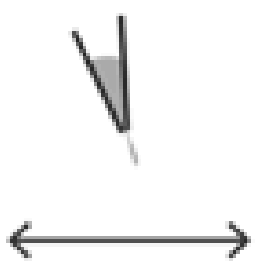

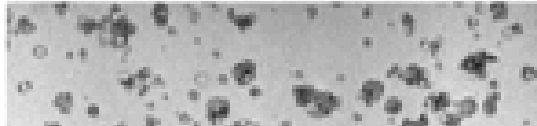

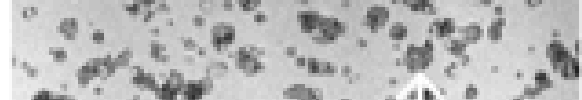
se 40 of is

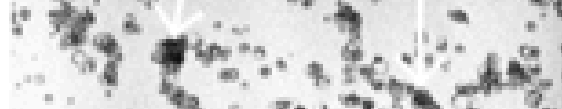

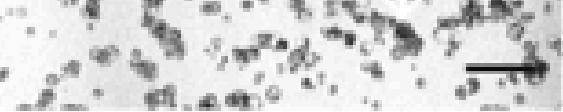

(F)

(E)

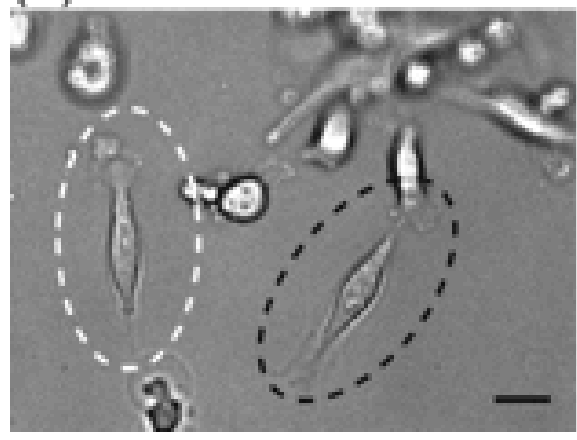

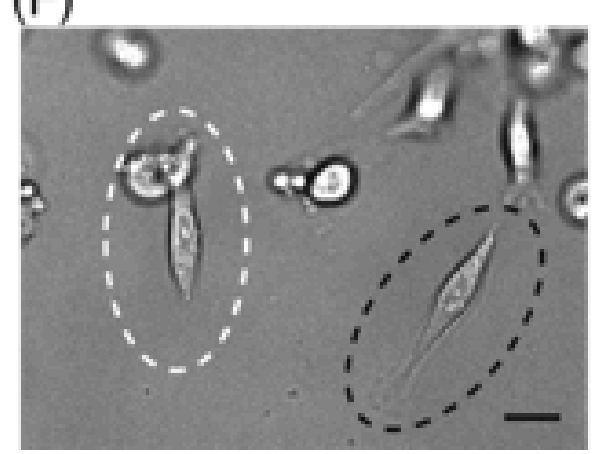

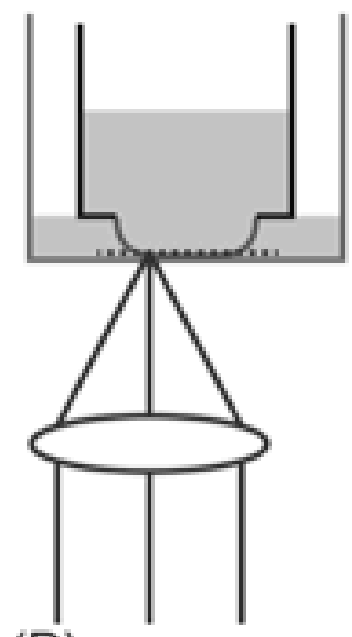

(D)

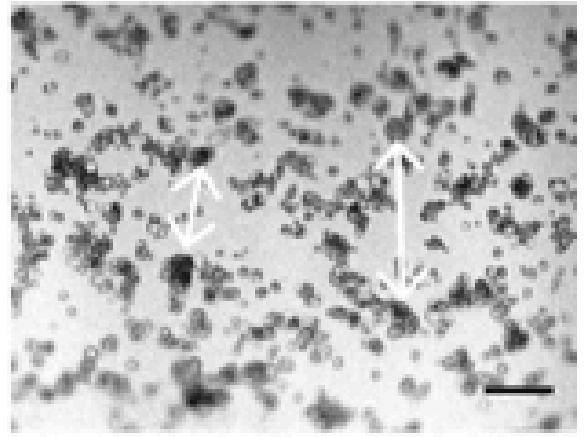

(G)

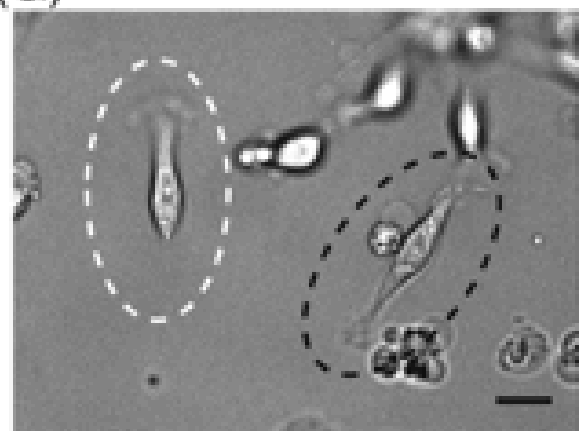




\section{Supporting Information}

\section{Characterization of thin gelatin hydrogel membranes with balloon properties for dynamic tissue engineering}

Morten Leth Jepsen, Line Hagner Nielsen, Anja Boisen, Kristoffer Almdal, Martin Dufva*

Figure S1. Technical drawings of 3D prints. a) Technical drawing of prints used for stretch with fluids. Measurements in mm. b) Technical drawing of prints used for stretch with air. Measurements in mm. c) Technical drawing of prints used for stretching of cell cultures. Measurements in $\mathrm{mm}$.

Figure S2. Principle sketch of casting of gelatin hydrogel membrane. a) $200 \mu \mathrm{L}$ gelatin and mTG mixture is pipetted onto a surface. b) 3D print for casting gelatin hydrogel membrane is dipped in the gelatin and mTG mixture. c) Resulting in a thin membrane in which seeding of cells is possible.

Figure S3. Casting of gelatin hydrogel membrane in other shapes and sizes. a) Gelatin hydrogel membrane casted in a circle of $18 \mathrm{~mm}$ in diameter. b) Stretch of gelatin hydrogel membrane in 18 $\mathrm{mm}$ diameter circle by $3 \mathrm{~mL}$ PBS in a beaker with PBS. c) Gelatin hydrogel membrane casted in a square with $16 \mathrm{~mm}$ sides. d) Stretch of gelatin hydrogel membrane in $16 \mathrm{~mm}$ sided square by PBS and food dye in a beaker with PBS. e) Stretch of gelatin hydrogel membrane in $16 \mathrm{~mm}$ sided square by PBS and food dye. f) Stretch of $2.5 \%$ gelatin hydrogel membrane.

Figure S4. SEM of dried stretched hydrogels. a) Gelatin hydrogel membrane stretched with air pressure. b) Gelatin hydrogel membrane stretched with air pressure after 1 hour. c) Gelatin hydrogel membrane stretched with air pressure after 66 hours. d) SEM on dried stretched hydrogel. Scale bar: $500 \mu \mathrm{m}$. e) SEM on dried unstretched hydrogel. Scale bar: $500 \mu \mathrm{m}$

Figure S5. Circular fitting in ImageJ used for determining the surface area of the stretched gelatin hydrogel membrane.

Figure S6. a) Time course of storage modulus measurements with a rheometer. b) Pressure as a function of stretch area ratio ( $7.5 \%$ gelatin: $n=5$ (red), $5 \%$ gelatin: $n=9$ (black). 
a

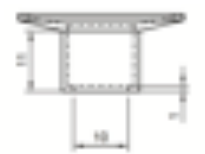

( ) b

$=\frac{\text { ind }}{L=1}$

国

() c

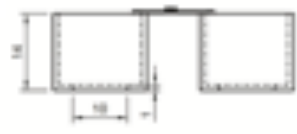

$+\cdots$<smiles>c1ccc2c(c1)C1c3ccccc3C2c2ccccc21</smiles> 
a

$V$

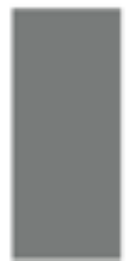

b

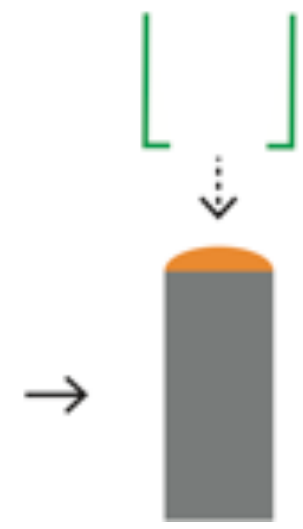

c

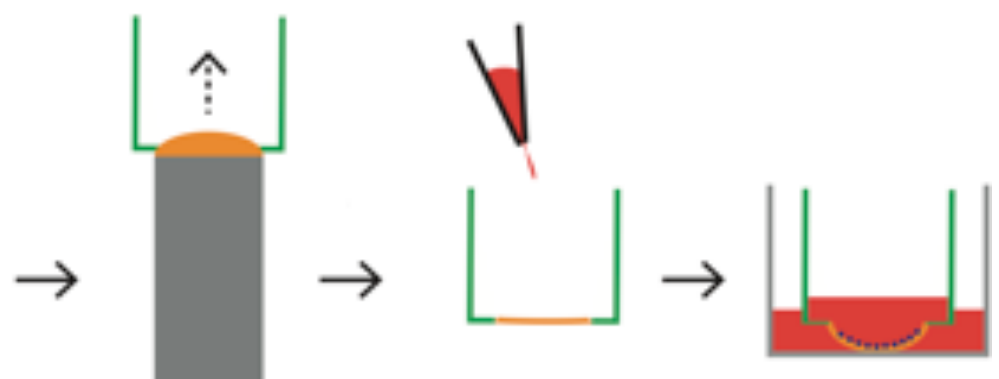




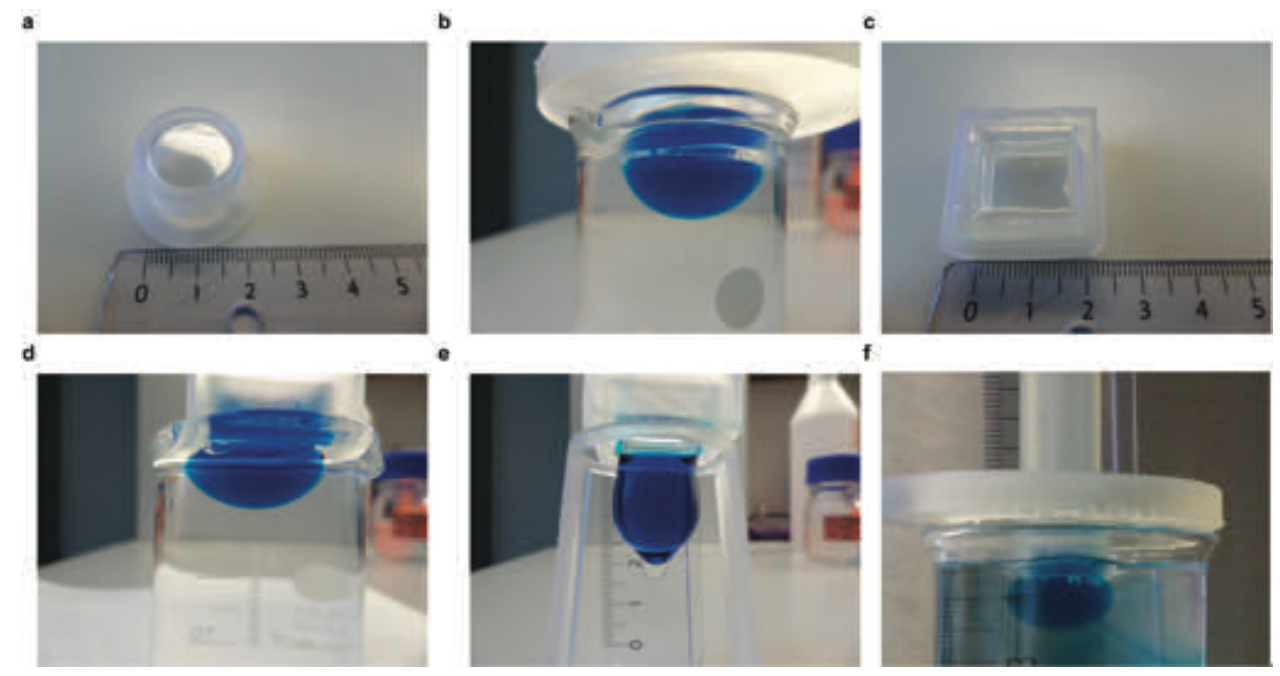


a

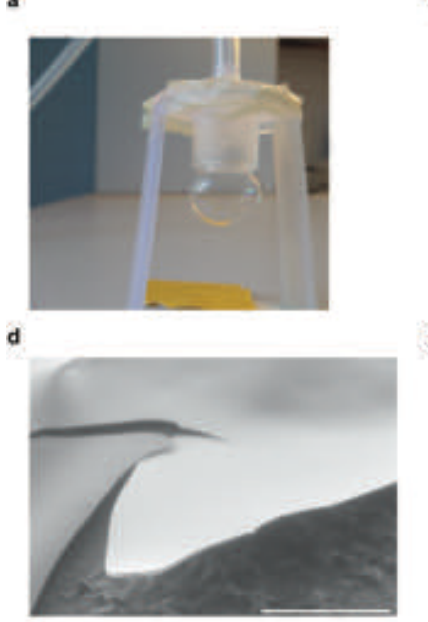

b

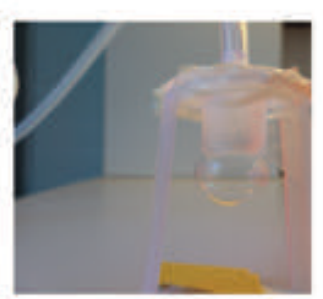

e

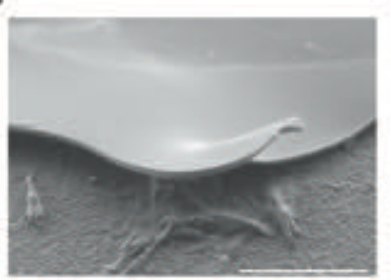

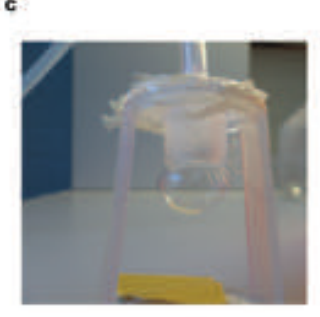




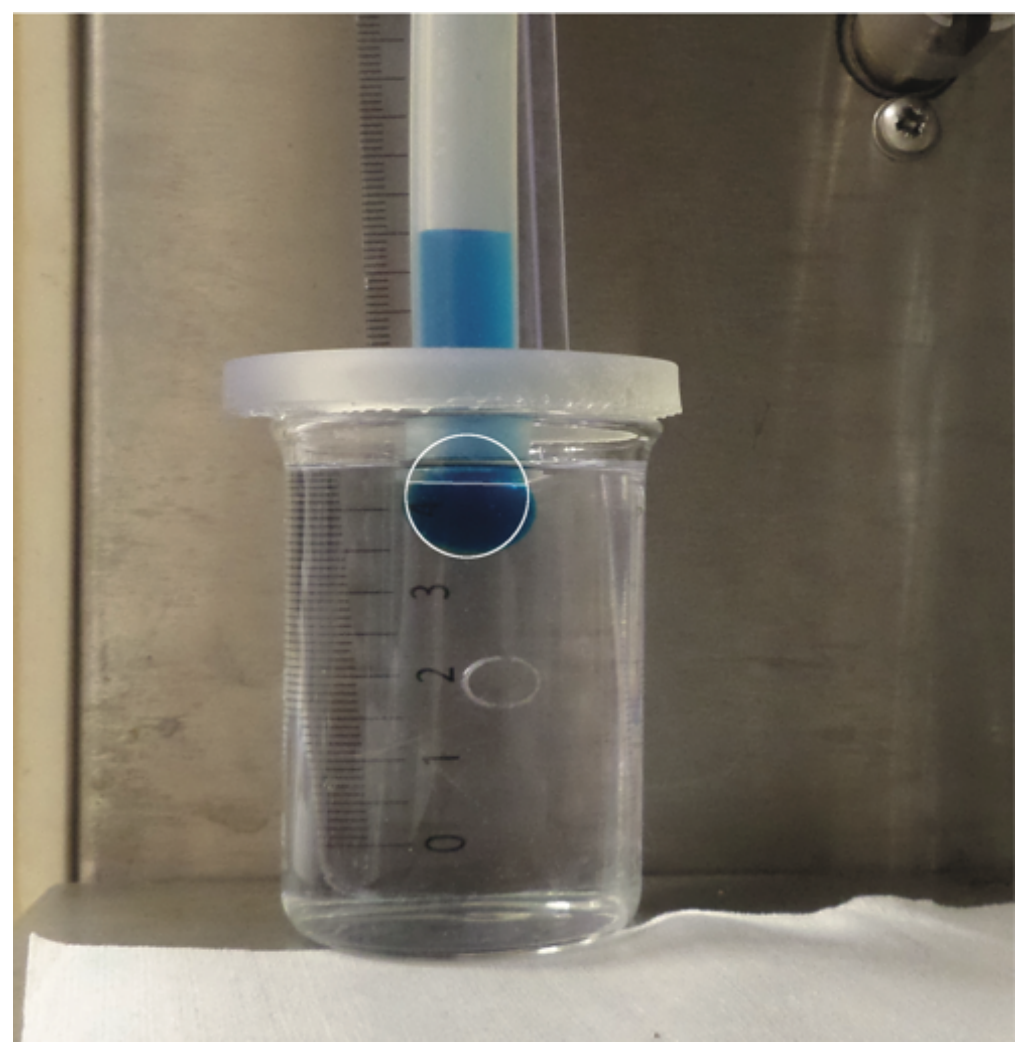


